\title{
Inteligencia emocional percibida en estudiantes de educación superior: análisis de las diferencias en las distintas dimensiones
}

\section{Perceived Emotional Intelligence in Higher Education Students: Analysis of Differences in the Different Dimensions}

\author{
Nieves Gutiérrez Ángel ${ }^{1}$ \\ ${ }^{1}$ Departamento de Educación, Universidad de Almería, España
}

Resumen. Objetivo. La finalidad es analizar la inteligencia emocional percibida en estudiantes universitarios. Método. Se trata de un diseño metodológico correlacional correspondiente a un diseño ex post facto, de carácter retrospectivo y comparativo. La muestra está compuesta por un total de 175 sujetos de tercer y cuarto curso del Grado de Educación Social. Resultados. Se muestra la existencia de diferencias en la inteligencia emocional atendiendo al sexo en cuanto a la atención y la regulación emocional, en las que las mujeres poseen puntuaciones más altas. En el caso de la edad, también existen diferencias estadísticamente significativas en cuanto a la regulación emocional, en la que obtienen puntuaciones más altas el alumnado cuyas edades se sitúan hasta los 20 años.

Palabras clave. Inteligencia emocional, inteligencia emocional percibida, edad, género, universitarios.

Abstract. Objective: The purpose is to analyze the emotional intelligence perceived in university students. Method: This is a correlational methodological design corresponding to an ex post facto design of retrospective and comparative. Participants: The sample is made up of a total of 175 subjects in the third and fourth year of a Social Education Degree. Results: This work shows the existence of differences in emotional intelligence based on sex in terms of attention and emotional regulation, in which women have higher scores. In the case of age, there are also statistically significant differences in terms of emotional regulation, in which students whose ages are up to 20 obtain higher scores.

Keywords. Emotional intelligence, perceived emotional intelligence, age, gender, university.

${ }^{1}$ Nieves Gutiérrez Ángel. Departamento de Educación, Universidad de Almería. Calle Universidad de Almería S/N. Código Postal: 04120. La Cañada, Almería. España. E-mail: nga212@ual.es

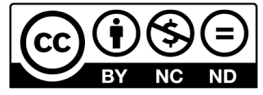

Esta obra está bajo una licencia de Creative Commons Reconocimiento-NoComercial-SinObraDerivada 4.0 Internacional. 


\section{Introducción}

El modelo de habilidad de la inteligencia emocional comprende el concepto como la suma de distintas habilidades cognitivas de cara a detectar, emplear, discernir y dominar las emociones (Mayer \& Salovey, 1997), tanto a nivel intrapersonal como interpersonal (Mayer, Salovey, \& Caruso, 2004). Siguiendo con este modelo, la inteligencia emocional se compone de cuatro secciones o ramas, integradas cada una de ellas por distintas habilidades, las cuales se desarrollan de forma diferente, atendiendo a su propia complejidad (Salovey, DetweilerBedell, Detweiler-Bedell, \& Mayer, 2008). En concreto, se corresponden con una estructura jerárquica, las cuales hacen alusión, de mayor a menor complejidad, a la regulación reflexiva de las emociones, la comprensión y análisis de las emociones, la facilitación emocional del pensamiento y la percepción, valoración y expresión de las emociones (Cabello, FernándezBerrocal, Ruiz, \& Extremera, 2006; Fernández-Berrocal \& Extremera, 2005; García del Castillo-López, García del Castillo, \& Marzo, 2013).

Por ello, el conjunto de todas estas habilidades se corresponde con el concepto global de inteligencia emocional, en el que el protagonismo recae en las habilidades para reflexionar en cuanto a las emociones, el potencial de estas para beneficiar y dirigir el pensamiento (Mayer \& Salovey, 1997), en la que no tiene cabida ningún aspecto de la personalidad (Fernández-Berrocal \& Extremera, 2009).

Conviene aclarar que, en la actualidad, se corresponde con uno de los modelos que más aceptación, difusión e investigación ha suscitado en España (Extremera \& FernándezBerrocal, 2002; Fernández-Berrocal, Extremera, \& Ramos, 2004; Fernández-Berrocal, Ramos, \& Extremera, 2001; Fernández-Berrocal, Salovey, Vera, Extremera, \& Ramos, 2005; Latorre \& Montañés, 2004; Limonero, Tomás-Sábado, Fernández-Castro, \& GómezBenito, 2004). Además, se trata del modelo que más soporte empírico y rigor científico posee (Cazalla-Luna \& Molero, 2016; Fernández-Berrocal \& Ramos, 1999; Mayer, Caruso, \& Salovey, 1999; Salguero, Fernández-Berrocal, \& Iruarrizaga, 2004).

Aunado a ello, este modelo posee, como una de sus más notorias contribuciones, el hecho de aportar un concepto de inteligencia emocional que puede ser evaluado a través de distintos instrumentos elaborados concretamente para ello; sobre todo, los test de ejecución, cuya finalidad reside en evaluar tanto la percepción de las propias habilidades emocionales como el nivel de ejecución de estas (Extremera, Salguero, FernándezBerrocal, \& Ruiz-Aranda, 2009; Salguero, Fernández-Berrocal, Ruiz-Aranda, Castillo, \& Palomera, 2011), como es el Trait Meta-Mood Scale-48 (TMMS-48) creado por Salovey, Mayer, Goldman, Turvey y Palfai (1995).

A partir de este modelo, autores como Aritzeta et al. (2016) identifican a la inteligencia emocional con tres dimensiones, creando un submodelo que, en este caso, se corresponde con la percepción, la comprensión y la regulación de las emociones. Siguiendo con la utilización de las medidas de autoinforme, los autores proponen la evaluación de la inteligencia emocional a través del TMMS-24 (Fernández-Berrocal et al., 2004), que se trata de un instrumento de evaluación elaborado a partir del Trait Meta-MoodScale (TMMS).

En los útlimos años, el estudio de la inteligencia emocional desde los contextos sociales y educativos ha cobrado un especial interes (Yudes, Peña, \& Pacheco, 2019) y se ha 
consolidado en estos ámbitos como una línea de investigación emergente, pues tales habilidades emocionales juegan un papel crucial ante el abordaje y afrontamiento de circustancias de carácter negativo (Ruvalcaba, Gallegos, Solis, Gabriela, \& Bravo, 2019).

Es decir, una persona considerada emocionalmente inteligente posee las habilidades necesarias para afrontar diversas situaciones estresantes con más éxito que aquella que no lo es. Siendo capaz, a su vez, de recibir y analizar los estados de ánimo propios y ajenos, expresarlos con claridad, regular sus propias emociones y emitir una respuesta de manera asertiva ante la situación estresante (Omar, Paris, Uribe, Ameida, \& Aguiar, 2011). En este sentido, cobra también una especial relevancia la implicación educativa y junto con ella la denominada educación emocional, la cual tiene como finalidad máxima el desarrollo de diversas competencias emocionales (Bisquerra \& Pérez-Escoda, 2007).

En el ámbito educativo universitario esta enseñanza de las competencias emocionales también se ha consolidado como un tema de gran relevancia (Singer, Guzmán, \& Donoso, 2009), ya que las habilidades emocionales son relevantes tanto para el desarrollo exitoso de un trabajo como para la inserción laboral (Matus \& Gutiérrez, 2015). Es decir, que mediante la educación emocional se está dotando al alumnado universitario de determinadas competencias muy valoradas en el mundo laboral, como son la capacidad para establecer interrelaciones positivas con los compañeros, la capacidad de resolución de incidentes, el trabajo cooperativo, la apertura y deseo al cambio o el pensamiento basado en la innovación (Luy-Montejo, 2019).

Además de ello, la etapa universitaria está caracterizada por ser un momento en el que el sujeto debe enfrentarse a numerosos retos que, en ocasiones, pueden percibirse por los propios estudiantes inmersos en esta etapa como estresantes para ellos (Saddki, Sukerman, \& Mohamad, 2017). Como consecuencia, estos pueden llegar a provocar en el alumnado problemas de salud, en general, y psicológicos, en particular (Bergin \& Pakenham, 2015; Storrie, Ahern, \& Tuckett, 2010). Ante lo cual, la inteligencia emocional se consolida como una herramienta adecuada para hacer frente a tales sucesos estresantes, obtener un adecuado rendimiento académico y conseguir el bienestar emocional (Parhiala et al., 2018; Villanueva, Montoya-Castilla, \& Prado-Gascó, 2017).

Sin embargo, los beneficios de unos adecuados niveles de inteligencia emocional no solamente son aplicables a esta etapa, sino que una vez en ejercicio, cuando sean profesores, la Inteligencia Emocional se considera un recurso a la hora de percibir, analizar y regular las emociones negativas (Suárez \& Martín, 2019) y un factor preventivo del malestar docente y laboral, conocido como síndrome de burnout, el cual se caracteriza por el cansancio emocional, la actitud apática y el sentimiento de falta de realización laboral (Maslach \& Jackson, 1986).

A esto se suma que las personas con altos niveles de inteligencia emocional se caracterizan por el empleo de un razonamiento de tipo flexible y el gusto por lo variado y flexible (Mayer \& Salovey, 1997; Jiménez \& López-Zafra, 2013) rasgos que propician una mayor comprensión y actitudes de ayuda hacia los demás, cuestiones ineludibles en el profesorado y que repercuten incluso en una mejora de la integración social (Buenrostro et al., 2012), finalidad máxima de todo educador social. 
En definitiva, la inteligencia emocional promueve el afrontamiento del estrés de manera eficiente, mejora el rendimiento académico, promueve el bienestar emocional e, incluso, el éxito profesional (Wang, Xie, \& Cui, 2016; Ordóñez, González, Montoya-Castilla, \& Schoeps, 2014). Por todo ello, la finalidad de este trabajo es analizar la inteligencia emocional percibida de los estudiantes universitarios. Las hipótesis de las que parte este trabajo son:

- Las mujeres presentan mayores niveles de inteligencia emocional (Mestre, Samper, Frías, \& Tur, 2009; Toussaint \& Webb, 2005).

- La edad no ejerce influencia en los niveles de inteligencia emocional, al igual que ocurre con el curso en que se encuentre el alumando (Shipley, Jackson, \& Segrest, 2010).

\section{Método}

El método empleado es identificado como correlacional y de ex post facto, de carácter retrospectivo y comparativo, ya que mediante el mismo se contrastan dos o más grupos según una determinada característica (eg: curso, sexo, titulación) con una variable dependiente. En este caso, se corresponden con las puntuaciones alcanzadas en la inteligencia emocional percibida obtenidos tras la aplicación del TMMS-24.

\section{Participantes}

La muestra está compuesta por un total de 175 sujetos que se encuentran realizando el tercer y cuarto curso de sus estudios de Grado; en concreto, el Grado de Educación Social de la Universidad de Almería (España), los cuales han sido seleccionados mediante un muestreo aleatorio simple. Antes de realizar el cuestionario, se ofreció a la muestra una breve descripción de la finalidad de la investigación por parte del investigador principal y se facilitó un documento de consentimiento informado que garantizaba la confidencialidad y el anonimato de los datos recabados en este trabajo, el cual fue leído y firmado por cada uno de los participantes y entregado junto con el cuestionario una vez realizado.

La edad media de la muestra es de 25.79 años, con una desviación típica $D T=8.01$. En cuanto al sexo, el 22.9\% $(n=40)$ son hombres, y el $77.1 \%(n=135)$ mujeres. En cuanto al curso, pertenecen al tercer curso el $42.9 \%(n=75)$ y al cuarto curso el $57.1 \%(n=100)$.

\section{Instrumentos}

Se han empleado dos instrumentos en este estudio. El primero de ellos, un instrumento elaborado ad hoc, cuyo objetivo fue recabar información de tipo sociodemográfico sobre la muestra, en cuanto a cuestiones como la edad, el sexo, la titulación o la formación en el contexto educativo.

El segundo instrumento empleado es el Trait Meta Mood Scale-24 (TMMS-24), elaborado por Fernández-Berrocal et al. (2004), el cual se corresponde con una adaptación del Trait Meta-MoodScale (TMMS), elaborado por Salovey et al. (1995), que posee como finalidad la evaluación de la inteligencia emocional percibida por el propio sujeto. Se trata de una medida de autoinforme de la inteligencia emocional, compuesto por veinticuatro ítems que evalúan las tres dimensiones de la inteligencia emocional aportadas por el modelo de Salovey y Mayer (1990): percepción, comprensión y regulación de las emociones. Posee ocho ítems que evalúan cada una de esas dimensiones (Rey \& Extremera, 2012; Rueda \& 
López, 2014) mediante una escala Likert de cinco puntos (en la que 1 significa nada de acuerdo y 5 significa el máximo de acuerdo con la afirmación) (Rey \& Extremera, 2012).

De tal manera, los ítems del uno al ocho evalúan la percepción emocional; del ítem nueve al dieciséis, evalúan la comprensión emocional; y, los ítems del diecisiete al veinticuatro evalúan la regulación emocional (Rueda \& López, 2014). En la actualidad, el TMMS24 se ha convertido en uno de los instrumentos más empleados a nivel nacional e como internacional, y es utilizado en investigaciones tanto de carácter educativo como psicológico (Extremera \& Fernández-Berrocal, 2005).

Las propiedades psicométricas de este instrumento son apropiadas (Fernández-Berrocal et al., 2004). En concreto, el $\alpha$ de Cronbach es de .90 para la percepción de las emociones, .90 para la comprensión de las emociones, $\mathrm{y}=.86$ para la regulación de las emociones (Cazalla-Luna, Ortega-Álvarez, \& Molero, 2015), al igual que posee una fiabilidad test-retest favorable, atención $=.60$; claridad $=.70$ y reparación $=.83$ (Extremera \& Fernández-Berrocal, 2005; Extremero, Fernández-Berrocal, Mestre, \& Guil, 2004). En el caso de este trabajo, $\alpha=.94$ para la atención emocional, $\alpha=.98$ para la comprensión de las emociones, $\mathrm{y} \alpha=.89$ para la regulación de las emociones.

\section{Procedimiento}

Se facilitó al alumnado, mediante el aula virtual de distintas asignaturas, un enlace en el que podían acceder a la información y realización del cuestionario. Previamente a ello, dicho cuestionario había sido creado en la plataforma de encuestas Lime Survey. El tiempo disponible para la realización del cuestionario fue ilimitado y la única restricción existente fue el hecho de que si no respondían todas las preguntas que aparecían en la pantalla, no les dejaría continuar. Además, también se configuró esta plataforma de encuestas para que en el caso de no acabarla en un intento, cuando volvieran a acceder, estuvieran guardadas las respuestas a las preguntas ya contestadas.

\section{Análisis de datos}

El análisis de datos se ha realizado mediante las pruebas $t$ de Student para grupos independientes; en este caso, aplicada con variables como el sexo o el curso, así como el análisis de la varianza de un factor (ANOVA), en este caso, con la edad. La realización de todas estas pruebas tuvo como finalidad no solamente medir las puntuaciones obtenidas en cuanto a la inteligencia emocional percibida, sino también analizar la posible existencia de diferencias entre cada grupo. Para finalizar, en aquellas situaciones en las que se encontraron diferencias estadísticamente significativas, se calculó la $d$ de Cohen (1988) para medir el tamaño del efecto. Todo ello a través de la utilización del programa de análisis estadístico SPSS en su versión 23.

\section{Resultados}

\section{Inteligencia emocional según el sexo}

En la Tabla 1 se muestran los índices estadísticos descriptivos para los dos grupos, en este caso, mujeres y hombres, en las tres dimensiones de la inteligencia emocional, atención, 
claridad y reparación emocional. Como se puede observar, las puntuaciones de las mujeres son más altas en todas estas dimensiones.

$\mathrm{Al}$ respecto, los resultados obtenidos a partir de la prueba $t$ para muestras independientes en función del sexo muestran diferencias estadísticamente significativas en atención emocional $\left(t_{(175)}=-3.19 ; p<.001 ; d=.55\right)$ y en regulación emocional $\left(t_{(175)}=-2.02 ; p<.05\right.$; $d=.36)$. Ante lo que se puede afirmar que las mujeres $(M=30.78)$ obtienen puntuaciones más elevadas que los hombres $(M=26.42)$; el tamaño del efecto es poco $(d<.20)$ o moderado $(d<.50)$.

\section{Inteligencia emocional según el curso}

En la Tabla 2 se muestran los índices estadísticos descriptivos para los dos grupos; en este caso, para tercer y cuarto curso. En las tres dimensiones de la inteligencia emocional, atención, claridad y reparación emocional. Como se puede observar, las puntuaciones del alumnado de tercer curso son más altas en todas las dimensiones.

Sin embargo, los resultados obtenidos a partir de la prueba $t$ para muestras independientes en función del curso, no muestran diferencias estadísticamente significativas en ninguna dimensión.

Tabla 1

Inteligencia emocional. Descriptivos y prueba t según sexo

\begin{tabular}{|c|c|c|c|c|c|c|c|c|c|}
\hline \multirow{2}{*}{ TMMS-24 } & \multicolumn{3}{|c|}{ Hombre } & \multicolumn{3}{|c|}{ Mujer } & \multirow{2}{*}{$t$} & \multirow{2}{*}{$p$} & \multirow{2}{*}{$d$} \\
\hline & $n$ & $M$ & $D T$ & $n$ & $M$ & $D T$ & & & \\
\hline $\begin{array}{l}\text { Atención } \\
\text { emocional }\end{array}$ & 40 & 26.42 & 8.30 & 125 & 30.78 & 7.25 & $-3.19^{* *}$ & .001 & .55 \\
\hline $\begin{array}{l}\text { Claridad } \\
\text { emocional }\end{array}$ & 40 & 29.32 & 8.64 & 125 & 29.87 & 7.96 & -3.70 & .71 & - \\
\hline $\begin{array}{l}\text { Reparación } \\
\text { emocional }\end{array}$ & 40 & 26.47 & 7.29 & 125 & 29.07 & 6.98 & $-2.02 *$ & .04 & .36 \\
\hline
\end{tabular}

Tabla 2

Inteligencia emocional. Descriptivos y prueba t según curso (tercero/ cuarto)

\begin{tabular}{|c|c|c|c|c|c|c|c|c|}
\hline \multirow{2}{*}{ TMMS-24 } & \multicolumn{3}{|c|}{ Educación Infantil } & \multicolumn{3}{|c|}{ Educación Primaria } & \multirow{2}{*}{$t$} & \multirow{2}{*}{$p$} \\
\hline & $n$ & $M$ & $D T$ & $n$ & $M$ & $D T$ & & \\
\hline $\begin{array}{l}\text { Atención } \\
\text { emocional }\end{array}$ & 35 & 31.85 & 6.20 & 123 & 29.26 & 8.22 & 1.72 & .08 \\
\hline $\begin{array}{l}\text { Claridad } \\
\text { emocional }\end{array}$ & 35 & 31.14 & 7.55 & 123 & 29.37 & 8.47 & 1.11 & .26 \\
\hline $\begin{array}{l}\text { Reparación } \\
\text { emocional }\end{array}$ & 35 & 29.85 & 6.73 & 123 & 27.72 & 7.24 & 1.56 & .12 \\
\hline
\end{tabular}




\section{Inteligencia Emocional según la edad}

En cuanto a la inteligencia emocional y la edad (Tabla 3), en primer lugar se crearon intervalos en cuanto a la edad. De tal manera, todos los grupos etáreos de la muestra quedaron agrupados en cuatro grupos de la siguiente manera: hasta los veinte años; edades comprendidas entre los 21 y los 29 años; edades comprendidas entre los 30 y los 39 años; y más de 40 años.

La Tabla 3 muestra los índices estadísticos descriptivos para cada una de las dimensiones de la inteligencia emocional en cuanto a la edad. Como se puede comprobar, el alumnado cuyas edades están comprendidas hasta los 20 años obtienen una media más alta que el resto, como es en el caso de la atención y la regulación emocional. A excepción del caso de la claridad emocional, en donde el grupo de edad que obtiene una media más alta se corresponde con los que sus edades están comprendidas entre los 30 y los 39 años. Los resultados de la prueba ANOVA de un factor manifiestan la existencia de diferencias estadísticamente significativas en la dimensión de regulación $(p<.001) F=10.13$.

Tabla 3

Anova entre la edad y la inteligencia emocional

\begin{tabular}{|c|c|c|c|c|c|c|}
\hline \multirow{2}{*}{$\begin{array}{c}\text { Dimensiones de la } \\
\text { inteligencia emocional }\end{array}$} & \multirow{2}{*}{ Grupos de edad } & \multirow{2}{*}{$n$} & \multirow{2}{*}{$M$} & \multirow{2}{*}{$D T$} & \multicolumn{2}{|c|}{ ANOVA } \\
\hline & & & & & $F$ & Sig. \\
\hline \multirow{4}{*}{ Atención emocional } & Hasta 20 años & 9 & 34.55 & 4.21 & \multirow{4}{*}{1.84} & \multirow{4}{*}{.13} \\
\hline & De 21 a 29 años & 129 & 29.23 & 7.93 & & \\
\hline & De 30 a 39 años & 19 & 29.42 & 7.14 & & \\
\hline & Más de 40 años & 8 & 33.00 & 7.01 & & \\
\hline \multirow{4}{*}{ Claridad emocional } & Hasta 20 años & 9 & 31.88 & 1.05 & \multirow{4}{*}{2.59} & \multirow{4}{*}{.05} \\
\hline & De 21 a 29 años & 129 & 28.83 & 8.50 & & \\
\hline & De 30 a 39 años & 19 & 33.47 & 6.11 & & \\
\hline & Más de 40 años & 8 & 33.00 & 7.01 & & \\
\hline \multirow{4}{*}{ Regulación emocional } & Hasta 20 años & 9 & 35.77 & 2.63 & \multirow{4}{*}{10.13} & \multirow{4}{*}{.001} \\
\hline & De 21 a 29 años & 129 & 26.98 & 7.00 & & \\
\hline & De 30 a 39 años & 19 & 33.42 & 4.65 & & \\
\hline & Más de 40 años & 8 & 31.87 & 6.26 & & \\
\hline
\end{tabular}

$* * * p<.001$ 


\section{Discusión}

Este trabajo se sitúa en la misma línea temática de otros que también han tomado como muestra a alumnando universitario (Cazalla-Luna \& Molero, 2016; Estrada, Monferrer, \& Moliner, 2016; Limonero, Gómez, Fernández-Castro, \& Tomás-Sábado, 2013; RosMorente, Mora, Nadal, Belled, \& Berenguer, 2017; Rueda \& López, 2014). Dentro de este colectivo, también destacan las investigaciones llevadas a cabo con alumnado universitario pertenecientes a las titulaciones de la rama de educación como Psicopedagogía, Educación Infantil, Educación Primaria, entre otras (Cazalla-Luna \& Molero, 2014; Cazalla-Luna et al., 2015; Suberviola-Ovejas, 2012). Además, también se encontraron distintas investigaciones realizadas en este caso con muestras compuestas por profesores tanto de educación primaria (Extremera, Durán, \& Rey, 2010; Pena, Rey, \& Extremera, 2012), como de secundaria (Pertegal-Felices, Castejón-Costa, \& Martínez, 2011) y ambos (De la Fuente, Franco, \& Salvador, 2010).

En el caso de este estudio, se puede afirmar que se trata de una mezcla de las investigaciones desarrolladas hasta el momento con este mismo instrumento, ya que por un lado, toma como muestra a estudiantes universitarios y, por el otro, escoge dentro de ese colectivo a estudiantes pertenecientes a titulaciones relacionadas con el mundo educativo; en este caso, con la educación social.

También, se debe mencionar que el presente estudio se enmarca dentro de aquellos que han estado relacionados con la evaluacuón de la inteligencia emocional, siendo destacables aquellos que han medido junto a la inteligencia emocional el género (Fernández-Berrocal \& Ruiz, 2008), la demanda empresarial (Pertegal-Felices et al., 2014), o el burnout (Sewell, 2011).

En cuanto a los resultados obtenidos, este trabajo pone de manifiesto la existencia de diferencias en cada uno de las dimensiones de la inteligencia emocional atendiendo al sexo. Resultados que coinciden con los aportados por otros trabajos (Del Rosal, Dávila, Sánchez, \& Bermejo, 2016; Páez \& Castaño, 2015; Valadaz, Borges, Ruvalcaba, Villegas, \& Lorenzo, 2013).

En el caso de este trabajo, son las mujeres las que obtienen puntuaciones más altas en todas las dimensiones, siendo estadísticamente significativas en el caso de la atención y la regulación emocional, resultados similares a los aportados por otros estudios (Extremera, Rey, \& Sánchez-Álvarez, 2019; Mestre \& Guil, 2003; Mestre, Samper, Frías, \& Tur, 2009; Riffle, 2010; Toussaint \& Webb, 2005). Por lo cual, se puede afirmar que la hipótesis 1 se cumple.

Los resultados obtenidos con respecto al sexo en este estudio son cuanto menos llamativos, pues se encontró, al respecto, que las mujeres obtienen puntuaciones estadísticamente significativas en lo que Mayer y Salovey (1997) consideran como las ramas más y menos complejas de la inteligencia emocional. Respectivamente, la atención emocional es la menos compleja de todas. Se trata de la capacidad tanto para la identificación, como para el reconocimiento de los sentimientos propios y ajenos. Conlleva, por tanto, el interés y el conocimiento de distintas señales acerca de la expresión, las sensaciones y la sinceridad de las emociones. 
Mientras que, por otro lado, la regulación emocional es la rama más compleja. Se trata de captar, analizar y reflexionar sobre las emociones, de cara al aprovechamiento y utilidad ellas, tanto de manera interpersonal, como intrapersonal. Con el objetivo principal de obtener a través de ello un desarrollo emocional e intelectual.

No obstante, si se aplican los criterios de corrección del propio instrumento (FernándezBerrocal et al., 2004), se puede concluir en el hecho de que tanto mujeres como hombres obtienen puntuaciones consideradas como adecuadas en cada una de las dimensiones que componen la inteligencia emocional.

En cuanto al curso, no existieron diferencias estadísticamente significativas entre el alumnado que pertenecía al tercer y cuarto curso, aunque el alumnado de tercer curso obtuvo puntuaciones más altas en todas las dimensiones. Por lo que, ante este hecho, se decidió analizar la posible influencia que la edad podría estar ejerciendo en los distintos niveles de inteligencia emocional.

Por ello, en tercer lugar, tomando en este momento como referencia a la variable edad, los resultados ponen de manifiesto la existencia de diferencias estadísticamente significativas en cuanto a la regulación emocional, dimensión en la que la muestra cuyas edades están comprendidas hasta los 20 años obtienen puntuaciones más altas, al igual que sucedía en otros trabajos (Riffle, 2010; Sanmartín, Gonzálvez, \& Vicent, 2018); por ello, la hipótesis 2 no se cumple.

Al respecto, destacan estudios como el elaborado por Shipley et al. (2010), quienes ponen de manifiesto la no existencia de relación entre la edad y los niveles en cada una de las dimensiones de la inteligencia emocional. Mientras que, por el contrario, estudios como el elaborado por Beadle et al. (2012) o el de Grühn, Rebucal, Diehl, Lumley y Labouvie-Vief (2008) resaltan el hecho de que, a medida que aumenta la edad, disminuye la inteligencia emocional.

Las aportaciones de este trabajo apoyan la idea de que, en la actualidad, el TMMS-24 es el instrumento de evaluación de la inteligencia emocional que mayor número de investigaciones posee en el terreno educativo (Aguilar-Luzon, Calvo-Salguero, \& Salinas, 2014; Casas, Ortega-Ruiz, \& del Rey, 2015).

No obstante, a pesar de estos resultados, es destacable que, dentro de este terreno, la mayoría de las investigaciones han tenido como finalidad el estudio de la relación existente entre la inteligencia emocional y el rendimiento escolar (Billings, Downey, Lomas, Lloyd, \& Stough, 2014; Brouzos, Misailidi, \& Hadjimattheou, 2014; Pulido \& Herrera, 2015), su relación con el comportamiento (Piqueras, Espinosa-Fernández, García-López, \& Beidel, 2012), así como también hay estudios centrados en el bullying (Aroca, Ros, \& Varela, 2016; Bartrina, 2014; Elipe, Ortega, Hunter, \& del Rey, 2012; Gázquez, Pérez-Fuentes, DíazHerrero, García-Fernández, \& Inglés, 2015; Ramos-Díaz, Jiménez-Jiménez, RodríguezFernández, Fernández-Zabala, \& Axpe, 2017).

Por lo anterior, futuras líneas de investigación deberían considerar algunos de los aspectos antes aludidos en relación con el alumnado universitario; no se debe olvidar que estas etapas evolutivas son de las más relevantes a lo largo del desarrollo vital, debido 
fundamentalmente a que es un periodo determinante en el proceso de construccion y consolidacion de la personalidad. A su vez, en esta etapa se forjan los cimientos emocionales de cara a la edad adulta, en cuanto a los comportamientos de carácter prosocial, el autoconocimiento y el sistema de valores propio (Eisenberg, Zhou, \& Koller, 2001; Koopmann-Holm \& Tsai, 2014).

Una de las grandes repercusiones de este estudio se sitúa, precisamente, en la hipótesis de que la educación universitaria debe promover el empleo y la capacitación de los futuros profesionales de la educación, para atender a las necesidades de la sociedad de manera general (González \& Wagenaar, 2003). Por ello, conocer sus niveles de inteligencia emocional será el primer paso para a partir de su conocimiento intervenir sobre dichas habilidades emocionales.

Desde esta investigación, se apuesta no solamente por la medición de la inteligencia emocional percibida, sino tambien por la importancia de desarrollar programas de educacion emocional de cara a mejorar la calidad de vida y el bienestar de los estudiantes universitarios (Mercer, Jani, Maxwell, Wong, \& Watt., 2012; Oladipo, Adenaike, Adejuno, \& Ojewurni, 2013). No puede olvidarse que los profesionales de la educación (como maestros o educadores sociales) son los principales responsables de las emociones de su alumnado (Fernández-Berrocal \& Extremera, 2002), por lo que se hace indispensable la implicación de tales profesionales para con la educación emocional, considerada como uno de los pilares del bienestar, adaptación y rendimiento óptimo del alumnado. En definitiva, es de especial interés el tratamiento de estos temas con los futuros profesionales de la educación, debido a que unos adecuados niveles de inteligencia emocional se traducirán en un mayor éxitos a la hora de afrontar situaciones negativas o de estrés a las que se enfrentan estos profesionales en su día a día (Extremera \& Fernández-Berrocal, 2004).

En cuanto a las limitaciones de este estudio, la primera de ellas es el tamaño de la muestra, ya que es reducido teniendo en cuenta que se trata de la población universitaria. Otra de las limitaciones es que se trata de un estudio transversal, por lo que la información aportada por él está sujeta a los contextos y situaciones concretas en la recogida de datos. Por esta razón, en investigaciones futuras sería recomendable la recogida de información en distintos momentos como, por ejemplo, a lo largo de formación académica. Por último, otra de las limitaciones y propuestas futuras se relaciona con la temática del estrés en los docentes; por ello, sería muy interesante comparar estos resultados entre el profesorado en formación y distintos grupos de profesores ya formados.

Una vez identificadas las limitaciones, futuras investigaciones tendrán por finalidad descubrir si tanto el factor de la experiencia como el factor de la formación, producen cambios en los resultados. Para ello, se debe tener en cuenta un carácter más longitudinal, en el que se integren nuevos ítems relacionados con el estrés docente o la mejora a partir de la aplicación de un programa de educación emocional.

\section{Referencias}

Aguilar-Luzón, M. C., Calvo-Salguero. A., \& Salinas, J. M. (2014). Beliefs and environmental behavior: the moderating effect of emotional intelligence. Scandinavian Journal of Psychology, 55(6), 619-629. doi: 10.1111/sjop.12160

Actualidades en Psicología, 34(128), 2020, 17-33 
Aritzeta, A., Balluerka, N., Gorostiaga, A., Alonso-Arbiol, I., Haranburu, M., y Gartzia, L. (2016). Classroom emotional intelligence and its relationship with school performance. European Journal of Education and Psychology, 9(1), 1-8

Aroca, C., Ros, C., \& Varela, C. (2016). Programa para el contexto escolar de prevención de violencia en parejas adolescentes. Educar, 52(1), 11-31.

Bartrina, M. J. (2014). Conductas de ciberacoso en niños y adolescentes: Hay una salida con la educación y la conciencia social. Educar, 50(2), 383-400.

Beadle, J. N., Sheehan, A. H., Dahlben, B., \& Gutchess, A. H. (2012). Aging, empathy, and prosociality. Journals of Gerontology Series B: Psychological Sciences and Social Sciences, 70(2), 213-222.

Bergin, A. \& Pakenham, K. (2015). Law student stress: Relationships between academic demands, social isolation, career pressure, study/life imbalance and adjustment outcomes in law students. Psychiatry, Psychology and Law, 22(3), 388-406. doi: 10.1080/13218719.2014.960026

Billings, C., Downey, L. A., Lomas, J. E., Lloyd, J., \& Stough, C. (2014). Emotional Intelligence and scholastic achievement in pre-adolescent children. Personality and Individual Differences, 65, 14-18. doi: 10.1016/j.paid.2014.01.017

Bisquerra, R. \& Pérez-Escoda, N. (2007). Las competencias emocionales. Educación, 21(10), 61-82.

Brouzos, A., Misalidi, P., \& Hadjimatheou, A. (2014). Associations Between Emotional Intelligence, Socio-Emotional Adjustment, and Academic Achievement in Childhood: The Influence of Age. Canadian Journal of School Psychology, 29(2), 8399. doi: $10.1177 / 0829573514521976$

Buenrostro, A., Valadez, D., Soltero, R., Nava, G., Zambrano, R., \& García, A. (2012). Inteligencia emocional y rendimiento académico en adolescentes. Revista de Educación y Desarrollo, 20(1), 29-37.

Cabello, R., Fernández-Berrocal, P., Ruiz, D., \& Extremera, N. (2006). Una aproximación a la integración de diferentes medidas de regulación emocional. Ansiedad y Estrés, 12(2-3), 155-166.

Casas, J., Ortega-Ruiz, R., \& Del Rey, R. (2015). Bullying: The impact of teacher management and trait emotional intelligence. British Journal of Educational Psychology, 85(3), 407423. doi: $10.1111 /$ bjep.12082

Castaño, J. J., y Páez, M. L. (2014). Inteligencia emocional y rendimiento académico en estudiantes universitarios. Psicología desde el Caribe, 32(2), 268-285.

Cazalla-Luna, N., Ortega-Álvarez, F., \& Molero, D. (2015). Autoconcepto e inteligencia emocional de docentes en prácticas. Revista Electrónica de Investigación y Docencia, (14), 151-164.

Cazalla-Luna, N. \& Molero, D. (2014). Inteligencia emocional percibida, ansiedad y afectos en estudiantes universitarios. Revista Española de Orientación y Psicopedagogía, 25(3), 56-73. 
Cazalla-Luna, N. \& Molero, D. (2016). Inteligencia emocional percibida, disposición al optimismo-pesimismo, satisfacción vital y personalidad de docentes en su formación inicial. Revista de Investigación Educativa, 34(1), 241-258. doi: 10.6018/rie.34.1.220701

Cohen, J. (1988). Statistical power analysis for the behavioral sciences. Hillsdale, NJ: Erlbaum.

De la Fuente, M., Franco, C., \& Salvador, M. (2010). Efectos de un programa de meditación (mindfulness) en la medida de la alexitimia y las habilidades sociales. Psicothema, 22(3), 369-375.

Del Rosal, I., Dávila, M. A., Sánchez., S., y Bermejo, M. L. (2016). La inteligencia emocional en estudiantes universitarios: diferencias entre el grado de maestro en educación primaria y los grados en ciencias. International Journal of Developmental and Educational Psychology, 2(1), 51-61.

Eisenberg, N., Zhou, Q., \& Koller, S. (2001). Brazilian adolescents' prosocial moral judgment and behavior: Relations to sympathy, perspective taking, gender-role orientation, and demographic characteristics. Child Development, 72(2), 518-534. doi: $10.1111 / 1467-8624.00294$

Elipe, P., Ortega, R., Hunter, S. C., \& Del Rey, R. (2012). Inteligencia emocional percibida e implicación en diversos tipos de acoso escolar. Behavioral Psychology, 20(1), 169-181.

Estrada, M., Monferrer, D., \& Moliner, M. A. (2016). Entrenamiento de la inteligencia emocional en el contexto de la formación en ventas. Revista Española de Orientación y Psicopedagogía, 27(2), 61-79.

Extremera, N., Durán, A., \& Rey, L. (2010). Recursos personales, síndrome de estar quemado por el trabajo y sintomatología asociada al estrés en docentes de enseñanza primaria y secundaria. Ansiedad y estrés, 16(1), 47-60.

Extremera, N., \& Fernández-Berrocal, P. (2002). Relation of perceived emotional intelligence and health-related quality of life in middle-aged women. Psychological Reports, 91(1), 47-59. doi: 10.2466/pr0.2002.91.1.47

Extremera, N. \& Fernández-Berrocal, P. (2004). La importancia de desarrollar la inteligencia emocional en el profesorado. Revista Iberoamericana de Educación, 33,1-10.

Extremera, N. \& Fernández-Berrocal, P. (2005). Inteligencia emocional percibida y diferencias individuales en el meta-conocimiento de los estados emocionales: una revisión de los estudios con el TMMS. Ansiedad y estrés, 11(2-3), 101-122.

Extremera, N., Fernández-Berrocal, P., Mestre,J. M., \& Guil, R. (2004). Medidas de evaluación de la inteligencia emocional. Revista Latinoamericana de Psicología, 36(2), 209-228.

Extremera, N., Rey, L., \& Sanchez-Alvarez, N. (2019). Validation of the Spanish version of the Wong Law emotional intelligence scale (WLEIS-S). Psicothema, 31(1), 94-100.

Extremera, N., Salguero, J. M., Fernández-Berrocal, P., \& Ruiz-Aranda, D. (2009). Acercamientos actuales en la medición científica de la inteligencia emocional. Algunas implicaciones en el uso en el ámbito laboral. En P. Fernández-Berrocal, N. Extremera, R. Palomera, D. Ruiz-Aranda, J. M. Salguero, \& R. Cabello 
(Coords.), Avances en el estudio de la inteligencia emocional (pp. 175-199). Santander: Fundación Marcelino Botín.

Fernández-Berrocal, P. \& Extremera, N. (2002). La inteligencia emocional como una habilidad esencial en la escuela. Revista Iberoamericana de Educación, 29(1), 1-6. doi: $10.35362 /$ rie2912869

Fernández-Berrocal, P. \& Extremera, N. (2005). La inteligencia emocional y la educación de las emociones desde el Modelo de Mayer y Salovey. Revista Interuniversitaria de Formación del Profesorado, 19(3), 63-93.

Fernández-Berrocal, P. \& Extremera, N. (2009). La Inteligencia Emocional y el estudio de la felicidad. Revista Interuniversitaria de Formación del Profesorado, 66(23), 85-108.

Fernández-Berrocal, P., Extremera, N., \& Ramos, N. (2004). Validity and reliability of the Spanish modified version of the Trait Meta-Mood Scale. Psychological Reports, 94(3), 751-755. doi: 10.2466/pr0.94.3.751-755

Fernández-Berrocal, P., \& Ramos, N. (1999). Investigaciones empíricas en el ámbito de la inteligencia emocional. Ansiedad y Estrés, 5(2-3), 247-260.

Fernández-Berrocal, P., Ramos, N., \& Extremera, N. (2001). Inteligencia Emocional, supresión crónica de pensamientos y ajuste psicológico. Boletín de Psicología, (70), 79-95.

Fernández-Berrocal, P., \& Ruiz, D. (2008). Emotional intelligence in education. Revista Electrónica de Investigación Psicoeducativa, 15(2), 421-436.

Fernández-Berrocal, P., Salovey, P., Vera, A., Extremera, N., \& Ramos, N. (2005). Cultural influences on the relation between perceived emotional intelligence and depression. International Review of Social Psychology, 18(1), 91-107.

García del Castillo-López, A., García del Castillo, J. A., \& Marzo, J. C. (2013). La relevancia de la inteligencia emocional en la prevención del consumo de alcohol. Información Psicológica, 104, 100-111.

Gázquez, J. J., Pérez-Fuentes, M. C., Díaz-Herrero, A., García-Fernández, J. M., \& Inglés, C. (2015). Perfiles de inteligencia emocional y conducta social en adolescentes españoles. BehavioralPsychology: Revista Internacional de Psicología Clínica y de la Salud, 23(1), 141-160.

González, J., \& Wagenaar, R. (2003). Tuning Educational Structures in Europe. Informe final. Fase Uno. Bilbao, Spain: University of Deusto and University of Groningen.

Grühn, D., Rebucal, K., Diehl, M., Lumley, M., \& Labouvie-Vief, G. (2008). Empathy across the adult lifespan: Longitudinal and experience-sampling findings. Emotion, $8(6), 753$.

Jiménez, M. I., \& López-Zafra, E. L. (2013). Impacto de la Inteligencia Emocional percibida, acti- tudes sociales y expectativas del profesor en el rendimiento académico. Electronic Journal of Research in Educational Psychology, 11(1), 75-98.

Actualidades en Psicología, 34(128), 2020, 17-33 
Koopmann-Holm, B., \& Tsai, J. L. (2014). Focusing on the negative: Cultural differences in expressions of sympathy. Journal of Personality and Social Psychology, 107(6), 10921115. doi: $10.1037 / \mathrm{a} 0037684$

Latorre, J. M., \& Montañés, J. (2004). Ansiedad, inteligencia emocional y salud en la adolescencia. Ansiedad y Estrés, 10(1), 111-125.

Limonero, J. T., Gómez, M. J., Fernández-Castro, J., \& Tomás-Sábado, J. (2013). Influencia de la inteligencia emocional percibida y la impulsividad en el abuso de cánnabis en jóvenes. Ansiedad y estrés, 19(2-3), 223-234.

Limonero, J. T., Tomás-Sábado, J., Fernández-Castro, J., \& Gómez-Benito, J. (2004). Influencia de la inteligencia emocional percibida en el estrés laboral de enfermería. Ansiedad y Estrés, 10(1), 29-41.

Luy-Montejo, C. (2019). El Aprendizaje Basado en Problemas (ABP) en el desarrollo de la inteligencia emocional de estudiantes universitarios. Propósitos y Representaciones, 7(2), 353-383.

Maslach, C. \& Jackson, S. E. (1986). MBI: Maslach burnout inventory; manual research edition. California, USA: Consulting Psychologists Press.

Matus, O. \& Gutierrez, A. (2015). Habilidades Blandas: Una ventaja competitiva en la formación tecnológica. GINT Journal of Industrial Neo-Technologies, 32-40.

Mayer, J. D., Caruso, D., \& Salovey, P. (1999). Emotional intelligence meets traditional standards for an intelligence. Intelligence, 27(4), 267-298. doi: 10.1016/S0160-2896(99)00016-1

Mayer, J. D., Salovey, P., \& Caruso, D. (2004). Emotional Intelligence: Theory, findings and implications. Psychological Inquiry, 15(3), 197-215.

Mayer, J. D. \& Salovey, P. (1997). What is emotional intelligence? En P. Salovey \& D. J. Sluyter (Eds.), Emotional development and emotional intelligence: Implications for educators (pp. 3-31). New York: Basic Books.

Mercer, S. W., Jani, B. D., Maxwell, M., Wong, S. Y., \& Watt, G. C. (2012). Patient enablement requires physician empathy: a cross-sectional study of general practice consultations in areas of high and low socioeconomic deprivation in Scotland. BMC Family Practice, 8(13), 6-9.

Mestre, J. M. \& Guil, R. (2003). Inteligencia emocional. En E.G. Fernández-Abas-cal, M. P. Jiménez \& M. D. Martín (eds.). Emoción y motivación: La adaptación bumana (pp. 397-425). Madrid: Centro de Estudios Ramón Areces.

Mestre, M. V., Samper, P., Frías, M. D., \& Tur, A. M. (2009). Are Women More Empathetic than Men? A Longitudinal Study in Adolescence. The Spanish Journal of Psychology, 12(1), 76- 83. doi: 10.1017/S1138741600001499

Oladipo, S. E., Adenaike, F. A., Adejumo, A. O., \& Ojewumi, K. O. (2013). Psychological Predictors of Life Satisfaction among Undergraduates. Procedia Social and Behavioral Sciences, 82, 292-297. doi: 10.1016/j.sbspro.2013.06.263 
Omar, A., Paris, L., Uribe, H., Ameida, S. H., \& Aguiar, M. (2011). Un modelo explicativo de resiliencia en jóvenes y adolescentes. Psicología em Estudo, 16(2), 269-277. doi: 10.1590/S1413-73722011000200010

Ordóñez, A., González, R., Montoya-Castilla, I., \& Schoeps, K. (2014). Conciencia emocional, estados de ánimo y rendimiento académico. International Journal of developmental and Educational Psychology. INFAD Revista de Psicología, 1(6), 229-236.

Parhiala, P., Torppa, M., Vasalampi, K., Eklund, K., Poikkeus, A., \& Aro, T. (2018). Profiles of school motivation and emotional well-being among adolescents: Associations with math and reading performance. Learning and Individual Differences, 61, 196204. doi: 10.1016/j.lindif.2017.12.003

Pena, M., Rey, L., \& Extremera, N. (2012). Life Satisfaction and engagement in elementary and primary educators: Differences in emotional intelligence and gender. Revista de Psicodidáctica, 17(2), 341-358.

Pertegal-Felices, M. L., Castejón-Costa, J. L., \& Martínez, M. A. (2011). Competencias socioemocionales en el desarrollo profesional del maestro. Educación XX1, 14(2), 237-260.

Pertegal-Felices, M. L., Jimeno-Morenilla, A., \& Sánchez-Romero, J. L. (2011). Use of Discussion Boards as a Student-Centered. Methodology for Large Groups in Higher Education, 27(1), 178-186.

Pertegal-Felices, M. L., Castejón-Costa, J. L., y Jimeno-Morenilla, A. (2014). Differences between the personal, social and emotional profiles of teaching and computer engineering professionals and students. Studies in Higher Education, 39(7), 1185-1201.

Piqueras, J. A., Espinosa-Fernández, L., Garcia-Lopez, L. J., \& Beidel, D. C. (2012). Validación del "Inventario de Ansiedad y Fobia Social-Forma Breve" en jóvenes adultos españoles. Behavioral Psychology, 20(3), 505-528.

Pulido, F., \& Herrera, F. (2015). Miedo e inteligencia emocional en el contexto pluricultural de Ceuta. Anuario de Psicología, 45(2), 249-263.

Ramos-Díaz, E., Jiménez-Jiménez, V., Rodríguez-Fernández, A., Fernández-Zabala, A., \& Axpe, I. (2017). Entrenamiento de la inteligencia emocional en el caso de una adolescente víctima de maltrato infantil. Revista de Psicología Clínica con Niños y Adolescentes, 4(1), 17-24.

Rey, L., \& Extremera, N. (2012). Inteligencia emocional percibida, felicidad y estrategias distractoras en adolescentes. Boletín de Psicología, 104, 87-101.

Riffle, B. J. (2010). Emotional maturity of adolescents and adults in GED programs. (Tesis inédita de doctorado). Mississippi, EE. UU.: Universidad del Sur del Mississippi.

Ros-Morente, A., Mora, C. A., Nadal, C. T., Belled, A. B., \& Berenguer, N. J. (2017). An examination of the relationship between emotional intelligence, positive affect and character strengths and virtues. Anales de Psicología, 34(1), 63-67. doi: 10.6018/ analesps.34.1.262891 
Rueda, B. \& López, C. E. (2014). Música y programa de danza creativa como herramienta de expresión de emociones. RETOS, Nuevas tendencias en Educación física, deporte y recreación, (24), 141-148.

Ruvalcaba, N. A., Gallegos, J., Solís, O., Gabriela, M., \& Bravo, H. R. (2019). Validez predictiva de las competencias socioemocionales sobre la resiliencia en adolescentes mexicanos. Diversitas: Perspectivas en Psicología, 15(1), 89-101. doi: 10.15332/s1794-9998.2019.0015.07

Saddki, N., Sukerman, N., \& Mohamad, D. (2017). Association between Emotional Intelligence and Perceived Stress in Undergraduate Dental Students. The Malaysian Journal of Medical Sciences, 24(1), 59-68. doi: 10.21315/ mjms2017.24.1.7

Salguero, J. M., Fernández-Berrocal, P., \& Iruarrizaga, I. (2004). Inteligencia emocional: Desarrollo y evolución del concepto. Boletín de la Sociedad Española para el Estudio de la Ansiedad y el Estrés, 21, 13-27.

Salguero, J. M., Fernández-Berrocal, P., Ruiz-Aranda, D., Castillo, R., \& Palomera, R. (2011). Inteligencia emocional y ajuste psicosocial en la adolescencia: El papel de la percepción emocional. European Journal of Education and Psychology, 4(2), 143-152.

Salovey, P., Detweiler-Bedell, B. T., Detweiler-Bedell, J. B., \& Mayer, J. D. (2008). Emotional Intelligence. In M. Lewis, J. M. Haviland-Jones \& I. Feldman (Eds.), Handbook of Emotions (pp. 533-547). New York: The Guilford Press.

Salovey, P., Mayer, J. D., Goldman, S. L., Turvey, C., \& Palfai, T. P. (1995). Emotional attention, clarity, and repair: Exploring emotional intelligence using the Trait Meta-Mood Sacale. In J. K. Pennebaker (Eds.), Emotion, Disclosure, and Health (pp. 125-154). Washington: American Psychological Association.

Salovey, P. \& Mayer, J. D. (1990). Emotional intelligence. Imagination, Cognition, and Personality, 9(3), 185-211. doi: 10.2190/DUGG-P24E-52WK-6CDG

Sanmartín, R., Gonzálvez, C., \& Vicent, M. (2018). Inteligencia emocional en alumnado de formación profesional. Diferencias en función del curso, del género y de la edad. Educar, 54(1), 229-245. doi: 10.5565/rev/educar.797

Sewell, G. (2011). Cómo la Inteligencia Emocional puede Influir Positivamente. Military Review, 4, 2-7.

Shipley, N. L., Jackson, M. J., \& Segrest, S. L. (2010). The effects of emotional intelligence, age, work experience, and academic performance. Research in Higher Education Journal, 9, 1-18.

Singer, M., Guzmán, R., \& Donoso, P. (2009). Entrenando Competencias Blandas en Jóvenes (Proyecto No1080292). Chile: Pontificia Universidad Católica de Chile e INACAP.

Storrie, K., Ahern, K., y Tuckett, A. (2010). A systematic review: students with mental health problems a growing problem. International Journal of Nursing Practice, 16(1), 1-6. 
Suárez, M. J., \& Martín, J. D. (2019). Influencia del perfil sociodemográfico del profesorado universitario sobre la inteligencia emocional y el burnout. Educación XX1, 22(2), 93-117, doi: 10.5944/educXX1.22514

Suberviola-Ovejas, I. (2012). Auto-percepción del profesorado sobre su formación en educación emocional. Revista de Comunicación Vivat Academia, 14, 1154-1167. doi: 10.15178/va.2011.117E.1154-1167

Toussaint, L. \& Webb, J. R. (2005). Gender differences in the relationship between empathy and forgiveness. The Journal of Social Psychology, 145(6), 673-685. doi: 10.3200/ SOCP.145.6.673-686

Valadaz, M. D. L. D., Borges, M. Á., Ruvalcaba, N., Villegas, K., y Lorenzo, M. (2013). La inteligencia emocional y su relación con el género, el rendimiento académico y la capacidad intelectual del alumnado universitario. Electronic Journal of Research in Educational Psychology, 11(2), 395-412.

Villanueva, L., Montoya-Castilla, I., \& Prado-Gascó, V. (2017). The importance of trait emotional intelligence and feelings in the prediction of perceived and biological stress in adolescents: Hierarchical regressions and fsQCA models. Stress, 20(4), 31-33. doi: 10.1080/10253890.2017.1340451

Wang, Y., Xie, G., \& Cui, X. (2016). Effects of Emotional Intelligence and Selfleadership on Students' Coping with Stress. Social Behavior and Personality, 44(5), 853-864. doi: 10.2224/sbp.2016.44.5.853

Yudes, C., Peña, L. R., \& Pacheco, N. E. (2019). Ciberagresión, adicción a internet e inteligencia emocional en adolescentes: un análisis de diferencias de género. Voces de la educación, [edición especial 2], 27-44. 